\title{
Electron-Pair Distribution in Chemical Bond Formation
}

\author{
M. Rodríguez-Mayorga, ${ }^{\dagger, \ddagger}$ M. Via-Nadal, ${ }^{\dagger}$ M. Solà, ${ }^{\ddagger}$ \\ J. M. Ugalde, ${ }^{\dagger}$ X. Lopez, ${ }^{\dagger}$ and E. Matito*,†, \\ †Kimika Fakultatea, Euskal Herriko Unibertsitatea, UPV/EHU, and Donostia International \\ Physics Center (DIPC). P.K. 1072, 20080 Donostia, Euskadi, Spain \\ $\ddagger$ Institut de Química Computacional i Catàlisi (IQCC) and Departament de Química, \\ University of Girona, C/ Maria Aurèlia Capmany, 69, 17003 Girona, Catalonia, Spain \\ ФIKERBASQUE, Basque Foundation for Science, 48013 Bilbao, Euskadi, Spain. \\ E-mail: ematito@gmail.com
}




\begin{abstract}
The chemical formation process from the study of radial intracule densities by constructing the relaxation holes, $\Delta h(u)$, resulting from the difference between the actual radial intracule density and the nonrelaxed one, which is obtained from atomic radial intracule densities and the pair density constructed from the overlap of the atomic densities has been studied. Our results show that the internal reorganization of electron pairs prior to bond formation and the covalent bond formation from electrons in separate atoms are completely recognizable processes from the shape of the relaxation hole, $\Delta h(u)$. The magnitude of $\Delta h(u)$, the shape of $\Delta h(u) \forall u<R_{e q}$ and the distance between the minimum and the maximum in $\Delta h(u)$ provide further information about the nature of the chemical bond formed.

A computational affordable approach to calculate the radial intracule density from approximate pair densities has been also suggested, paving the way to study electronpair distributions in larger systems.
\end{abstract}




\section{Graphical Abstract}

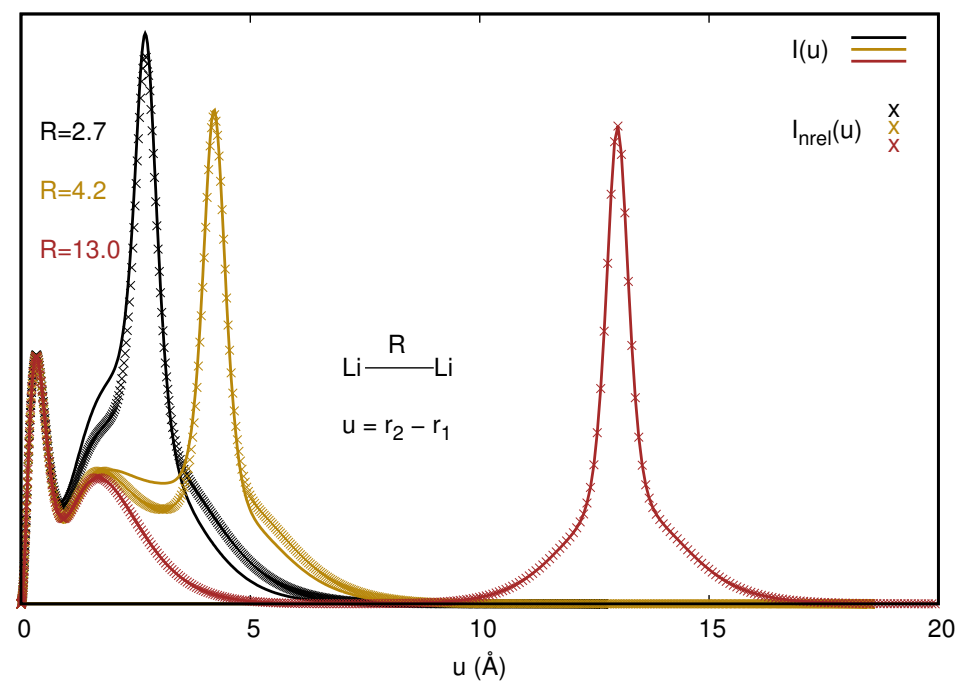

Figure 1: Relaxed and nonrelaxed radial intracule probability densities of $\mathrm{Li}_{2}$ at different bond lengths. All $R$ and $u$ in $\stackrel{\circ}{A}$. 


\section{Introduction}

Understanding chemical processes requires a proper characterization of bond formation. The formation of bonds is usually analyzed from the energy gain or loss, through the study of potential energy surfaces, ${ }^{1}$ suggested by René Marcelin in 1913. Since the advent of quantum mechanics, the chemical bond has been also investigated from descriptors based on the electron density. ${ }^{2-4}$ Many tools have been designed to this aim, the Quantum Theory of Atoms In Molecules ${ }^{5}$ (QTAIM) of Bader probably being the most popular one. Another avenue consists in the study of electron pair formation in chemical bond, as pionnered by the classical work of Lewis in $1916 .{ }^{6}$ Since the landmark paper of Lewis, there have been many attemps to fit the classic idea of electron pairs in the chemical bond within the framework of quantum mechanics, mostly using electron-pair distributions. ${ }^{7-21}$ The electron-pair distribution or pair density provides a quantum-mechanical description of the distribution of electron pairs in the space. ${ }^{22,23}$ Although the pair density has a simple probabilistic interpretation, it is a complicated six-coordinate function that is not easy to analyze. Most analysis of the pair density employ transformations that reduce the dimensionality, ${ }^{10,24,25}$ use statistical quantities such the average number of pairs, ${ }^{7-9,11-14,16,18,26,27}$ or employ two-electron expectation values such as the energy ${ }^{28-35}$ or the square of the total spin angular momentum. ${ }^{36-39}$ One of the most convenient transformations of the pair density is the so-called intracule density, which results from the integration of the pair density over the extracule coordinate. The radial or isotropic intracule density depends only on one coordinate, the interelectronic distance, but it still retains information about the electron-pair distribution and it is also the simplest quantity in terms of which an explicit expression of the electron-electron energy is known. Interestingly, the intracule density is related to an experimental observable, as it can be obtained from X-ray scattering techniques. ${ }^{40-42}$ The intracule density has been previously used to analyze the electronic structure and electron correlation of some molecular systems ${ }^{24,43-59}$ but very few studies of the intracule density have been devoted to the investigation of bond formation. ${ }^{60-62}$ 
The aim of this paper is to understand the changes occurring in the intracule density during the chemical bond formation. To this aim, we have chosen three simple molecules: $(i) \mathrm{H}_{2}$ and $(i i) \mathrm{HeH}^{+}$, which have a chemical bond consisting of a single electron pair, and (iii) BH, which contains several electron pairs, only one of which is involved in the chemical bond. The study is complemented with molecules presenting more complicated bonding situations such as $\mathrm{CO}, \mathrm{Li}_{2}, \mathrm{~F}_{2}$ or the ground and first excited states of $\mathrm{LiH}$. Finally, we consider the cost of the intracule density plots and suggest a means to obtain it at a reduced computational overhead.

\section{Methodology}

The pair probability density or simply pair density ${ }^{25,63}$ (2-PD) is defined as

$$
\rho_{2}\left(\mathbf{r}_{1}, \mathbf{r}_{2}\right)=\frac{N(N-1)}{2} \int\left|\Psi\left(\mathbf{r}_{1}, \mathbf{r}_{2}, \mathbf{r}_{3}, \ldots, \mathbf{r}_{N}\right)\right|^{2} d \mathbf{r}_{3} \ldots d \mathbf{r}_{N}
$$

for any electronic wavefunction $\Psi$ of a $N$-electron system. It is proportional to the probability of finding a pair of electrons at $\mathbf{r}_{1}$ and at $\mathbf{r}_{2}$, regardless the position of the other $N-2$ electrons. Among all electron-pair distributions, we may select the ones that satisfy $\mathbf{u}=$ $\mathbf{r}_{2}-\mathbf{r}_{1}$ for a fixed $\mathbf{u}$

$$
I(\mathbf{u})=\int \rho_{2}\left(\mathbf{r}_{1}, \mathbf{r}_{2}\right) \delta\left(\mathbf{u}-\mathbf{r}_{1}+\mathbf{r}_{2}\right) d \mathbf{r}_{1} d \mathbf{r}_{2}
$$

which is known as the intracule probability density or simply intracule density. Upon integration over the solid angle $\Omega_{u}$ we obtain the radial or isotropic intracule density,

$$
I(u)=u^{2} \int I(\mathbf{u}) d \Omega_{u}
$$

This function only depends on the interelectronic distance and, therefore, it provides a simple visualization of the distribution of electron-electron separations. By monitoring the changes of this distribution as we stretch a chemical bond, we should observe the formation and 
breaking of electron pairs and the electron reorganization in the molecule. Unfortunately, the radial intracule density contains all the information of the $N(N-1)$ electron pairs in the molecule, most of which is superflous to explain the chemical bond formation. In order to select the chemical important information within the radial intracule density, some of us ${ }^{62}$ defined the relaxation hole,

$$
\Delta h(u) \equiv \Delta h_{\text {rel }}(u)=I(u)-I_{\text {nrel }}(u),
$$

as the difference between the actual radial intracule density and the nonrelaxed one,

$$
I_{\text {nrel }}(u)=\sum_{A} I_{A}(u)+\sum_{A>B} I_{A B}(u)
$$

where the first term at the r.h.s. accounts for the atomic contribution and is computed from isolated atoms and the second term involves the summation of all interatomic contributions computed using nonrelaxed densities,

$$
I_{A B}(u)=u^{2} \int \rho_{A}\left(\mathbf{r}_{1}\right) \rho_{B}\left(\mathbf{r}_{2}\right) \delta\left(\mathbf{u}-\mathbf{r}_{1}+\mathbf{r}_{2}\right) d \mathbf{r}_{1} d \mathbf{r}_{2} d \Omega_{u}
$$

where $\rho_{A}\left(\mathbf{r}_{1}\right)$ is the density of the isolated atom $A$. The nonrelaxed density is thus the intracule density that can be obtained using atomic information only. Notice that the nonrelaxed density considers the distribution of electron pairs within the atoms but also the electron pairs generated from the two individual atomic densities, $\rho_{A} \rho_{B} . I_{\text {nrel }}(u)$ was proven to be a "poor man's approach to the real $I(u)$ " 62 and insufficient to characterize van der Waals interactions. ${ }^{54,62}$ However, the structure of $h_{\text {rel }}(u)$ was shown to provide valuable information about the bonding nature of small few-electron systems. ${ }^{62}$ In this work we are concerned with the bond formation process in species with larger number of electrons, which we will study through the analysis of $h_{\text {rel }}(u)$ at different bond lengths for various diatomic molecules. 
The computational cost of the intracule density is quite high because it involves the calculation of the second-order reduced density matrix (2-RDM) from a highly-accurate wavefunction (typically a full-configuration interaction, FCI) and the numerical integration with a Gauss-Hermite quadrature of Eq. 2 and a surface integration using a Lebedev quadrature (Eq. 3). In this paper we will consider two different approximations that can reduce the computational cost. First of all, we will substitute the FCI calculation with a sufficiently accurate wavefunction. Namely, we will consider coupled-cluster single and doubles (CCSD) and complete active space self-consistent field (CASSCF) wavefunctions as substitutes of the FCI calculation in the presence of dynamic and nondynamic correlation effects, respectively. ${ }^{64,65}$ Second, we will use an approximation of the 2-RDM that only includes two-index elements ${ }^{66,67}$ and, therefore, reduces the 2-RDM from the exact four-index quantity to an approximate two-index one.

CCSD wavefunctions do not satisfy the Hellmann-Feynman theorem and usually expensive energy-derivative 2-RDM are employed. ${ }^{35,68-70}$ In order to reduce the cost, several authors ${ }^{14,20,71-73}$ have used 2-PD approximations extracted from the reduced density matrix functional theory (RDMFT). ${ }^{53,74-76}$ In this paper we opt for the same solution to avoid the cost of CASSCF and CCSD 2-RDM. Among the different RDMFT approximations, we have chosen the simple Müller approximation ${ }^{66}$ (also known as Baerends-Buijse approximation ${ }^{67,77}$ ) that provides reliable results in the calculation of chemical bonding descriptors. ${ }^{14,20,53}$

FCI calculations of the potential energy curves (PECs) have been performed for $\mathrm{H}_{2}, \mathrm{HeH}^{+}$, $\mathrm{BH}, \mathrm{Li}_{2}$ and $\mathrm{LiH}$ (for $\mathrm{LiH}$ both ground and first excited states are considered) with a modified version of the code developed by Knowles and Handy. ${ }^{78,79}$ For $\mathrm{F}_{2}$ and CO, CASSCF calculations of the PEC were performed using Gaussian $09^{80}$ code taking ten electrons in six orbitals for $\mathrm{F}_{2}$ and six electrons in six orbitals, including a state average of six energy levels, for CO. Gaussian 09 package was employed to perform CCSD calculations for all diatomics but $\mathrm{CO}$ and two-electron molecules. The computation of approximate $\Delta h(u)$ using CCSD 
wavefunctions employed the unrestricted formalism in order to compute the total radial intracule density and the nonrelaxed ones (which often involve open-shell species) in the same grounds. All CCSD calculations included the correlation of all the electrons except for $\mathrm{F}_{2}$, for which we performed frozen-core calculations in order to produce a meaningful comparison between CCSD and CASSCF results. In all cases the aug-cc-pVDZ basis was used. PECs of all the studied systems are collected in Fig. S1. 2-RDM were produced from the expansion coefficients of CASSCF and FCI wave functions using the in-house DMN code. ${ }^{81,82}$ Intracule densities were computed with $R H O 2 \_O P S^{83}$ code using the algorithm of Cioslowski and Liu. $^{84}$

\section{Results}

\section{$3.1 \quad \mathrm{H}_{2}, \mathrm{HeH}^{+}$and $\mathrm{BH}$}

All the equilibrium distances at the corresponding level of theory are collected in Table 1 . The formation of the covalent bond in $\mathrm{H}_{2}$ is due to a partial deformation of the electron density of the two isolated atoms, which is relocated in between them. The same phenomenon can be studied in terms of the pair density, by comparing the radial intracule density of $\mathrm{H}_{2}$ to the nonrelaxed radial intracule density described in the previous section. The formation of an electron pair between the two atoms is evident from the plots in Fig. 2, where we observe that $\Delta h(u)$ peaks at the bond length or at shorter distances and is negative at larger distances. In other words, the electron-pair distance shrinks upon the formation of the chemical bond. The distance between the maximum and minimum of $\Delta h(u)$ provides information about the deformation of the electron-pairs length (see Table 1). In $\mathrm{H}_{2}$, this length systematically reduces as the molecule is formed, increasing the probability of having the electron pair at shorter distances. 

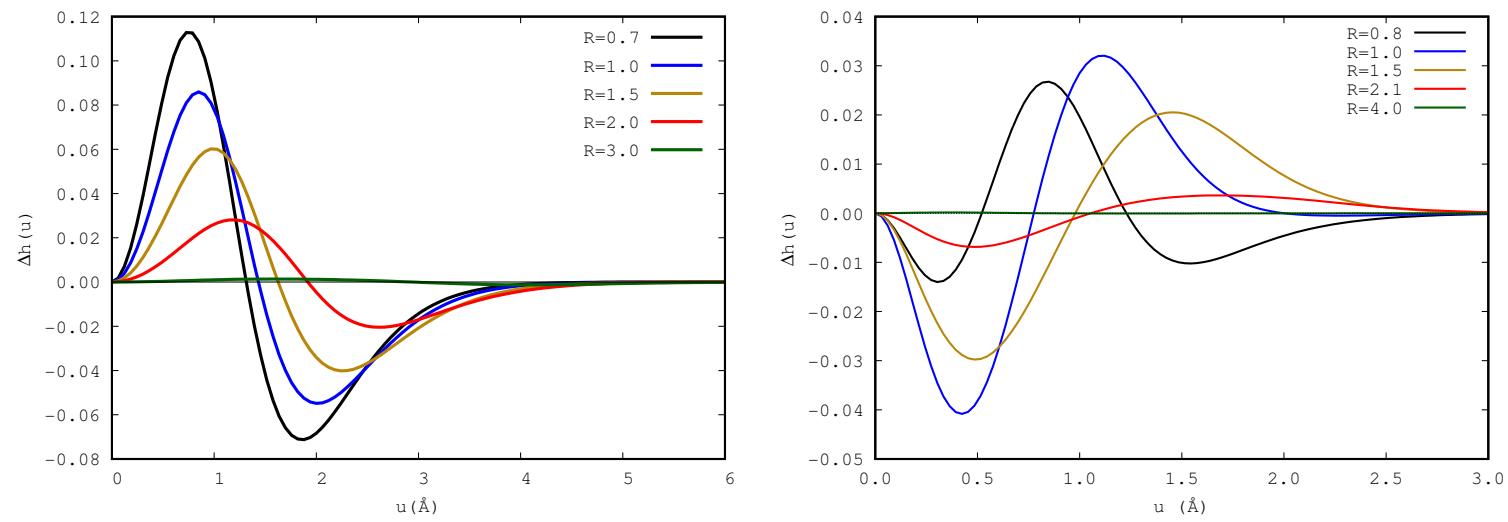

Figure 2: $\Delta h(u)$ of $\mathrm{H}_{2}$ (left) and $\mathrm{HeH}^{+}$(right) at different bond lengths. All $R$ and $u$ in $\stackrel{\circ}{A}$.

$\mathrm{HeH}^{+}$is formed from $\mathrm{He}$ and $\mathrm{H}^{+}$and, therefore, the nonrelaxed intracule density of this diatomic molecule is rather simple because $\mathrm{H}^{+}$does not contribute to atomic or diatomic components of the nonrelaxed intracule density (the density and the pair density of $\mathrm{H}^{+}$are zero). Hence, $\Delta h(u)$ only has contributions from the atomic He component and it consists in the difference of the radial intracule density of $\mathrm{HeH}^{+}$and He. The redistribution of the electron-pair probability density upon bond formation is less important than in the hydrogen molecule, as the values of $\Delta h(u)$ are one order of magnitude smaller. The formation of the covalent bond in this molecule is actually completely opposite to the latter case as we can see in Fig. 2. First, as the helium atom approaches the proton, the electron pair within He stretches, reducing the probability of having the electrons separated ca. $0.5 \AA$ and increasing it around the bond length. Only at the equilibrium, $R=0.8 \AA$, the electron-pair density at larger distances is reduced. The fact that the pair-density is dragged from short distances always around the same position (around $0.3-0.5 \AA$ ) is in accord with the fact that this molecule is just experiencing an internal pair reorganization of the electrons within He.

The formation of $\mathrm{BH}$ from $\mathrm{B}$ and $\mathrm{H}$ is an intermediate case where a bonding electron pair is formed from two electrons that come one from each atom, and there is simultaneously an internal reorganization of the electron pairs in B. At large atomic separations, as a result 
of the deformation of the electron density within $\mathrm{B}$ atom, electrons move towards $\mathrm{H}$ and, hence, the electron-pair distribution shifts to larger distances (see Fig. 3). Eventually, the two atoms get quite close and the profile of $\Delta h(u)$ reverses: the electron-pair distribution increases around the bond length by reducing the density of electron pairs at shorter and longer distances. Finally, the bond is completely formed and the peak of $\Delta h(u)$ is entirely due to the reduction of the distance of electron pairs. As we can see, the internal reorganization of electron pairs prior to bond formation and the covalent bond formation from electrons in separate atoms are completely recognizable processes from the shape of the relaxation hole.

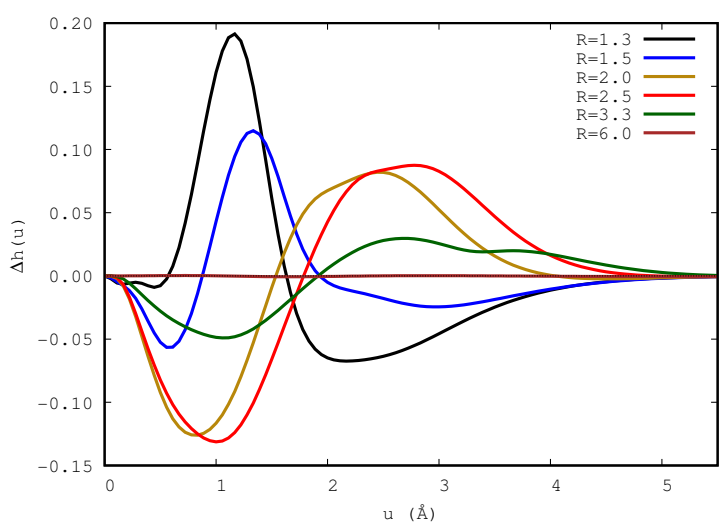

Figure 3: $\Delta h(u)$ of $\mathrm{BH}$ along the bond formation process. All $R$ and $u$ in $\AA$.

\section{$3.2 \quad \mathrm{Li}_{2}, \mathrm{CO}, \mathrm{F}_{2}$ and $\mathrm{LiH}$}

In this section we analyze the intracule densities during the bond formation of $\mathrm{Li}_{2}, \mathrm{CO}, \mathrm{F}_{2}$ and $\mathrm{LiH}$ from the neutral atoms in gas phase. In the latter case we study both the ground and the first excited states, $X^{1} \Sigma^{+}$and $A^{1} \Sigma^{+}$, respectively. All these diatomic molecules dissociate into neutral atoms in gas phase and there is, at least, some partial covalent character in the bonds of these molecules at equilibrium. ${ }^{85}$ 
Table 1: First maximum $(M)$ and minimum $(m)$ of $\Delta h(u)$ for all the systems studied. $u$ and $R_{e q}$, the equilibrium distance, in $\stackrel{\circ}{A}$.

\begin{tabular}{cccccc}
\hline Molecule & $R_{e q}$ & $u_{M}$ & $u_{m}$ & $\Delta h\left(u_{M}\right)$ & $\Delta h\left(u_{m}\right)$ \\
\hline $\mathrm{H}_{2}$ & 0.7 & 0.76 & 1.86 & 0.113 & -0.071 \\
$\mathrm{HeH}^{+}$ & 0.8 & 0.84 & 0.31 & 0.026 & -0.014 \\
$\mathrm{BH}$ & 1.3 & 1.16 & 2.17 & 0.192 & -0.067 \\
$\mathrm{Li}_{2}$ & 2.7 & 2.02 & 4.08 & 0.344 & -0.239 \\
$\mathrm{CO}$ & 1.1 & 0.69 & 1.19 & 1.287 & -1.038 \\
$\mathrm{~F}_{2}$ & 1.5 & 0.77 & 1.32 & 0.516 & -0.903 \\
$\mathrm{LiH}\left(X^{1} \Sigma^{+}\right)$ & 1.6 & 1.37 & 3.04 & 0.322 & -0.185 \\
$\mathrm{LiH}\left(A^{1} \Sigma^{+}\right)$ & 2.6 & 6.05 & 2.21 & 0.263 & -0.409 \\
\hline
\end{tabular}

In $\mathrm{F}_{2}$ the reorganization of electron pairs occurs faster than in $\mathrm{BH}$ (see Fig. 4). Indeed, the first appreciable variation of the electron pair distribution does not occur until the atoms are separated $2.5 \AA$, i.e. at $1.2 \AA$ from the equilibrium distance. The first significant values of $\Delta h(u)$ occur at $R=2.1 \stackrel{\AA}{ }$, where the electron-pair distribution is shifted to shorter distances, augmenting the probability of finding electron pairs between the $\mathrm{F}$ atoms. Unlike $\mathrm{H}_{2}$ or $\mathrm{BH}$, the maximum of the relaxation hole, $\Delta h(u)$, occurs at values of $u$ significantly shorter than the equilibrium distance (see Table 1), suggesting that two bonding electrons lie in the bonding region as opposed to the situation in which the electrons of the bonding pair are sitting close to the nuclei. The same situation is reproduced in $\mathrm{Li}_{2}$ and $\mathrm{CO}$ and, therefore, we are deemed to conclude that this profile is typical in covalent bonds. Obviously, $\mathrm{H}_{2}$ constitutes an exception because there are no core electrons in this molecule and, therefore, the electrons in the bonding pair are highly attracted towards the closest nucleus.

Let us now examine $\mathrm{Li}_{2}$, which presents a non-nuclear attraction (NNA) in the middle of the bond at various bond lengths $\left(R_{\mathrm{LiLi}} \in[2.7-3.3] \AA\right)^{86}$ and it is the smallest electride documented thus far. ${ }^{87}$ At the equilibrium, we find that $\Delta h(u)>0$ for all $u<R_{e q}$ and significant large $\Delta h(u)$ at $u=R_{e q} / 2$, indicating the additional formation of electron pairs 
between the electrons at the NNA and the ones at each Li atom. In this case, the formation of the molecule occurs less abruptly that in $\mathrm{F}_{2}$ and involves the reorganization of electron pairs at larger distances.

The $\Delta h(u)$ evolution with the bond length in $\mathrm{CO}$ also has some resemblance with the latter two cases. There are, however, three important differences. The first one is that the CO bond formation takes place in a shorter span than $\mathrm{Li}_{2}$ but larger than in $\mathrm{F}_{2}$, the first impor-

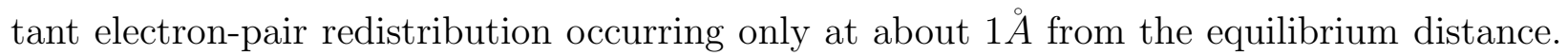
The second one is the long-range peaks of $\Delta h(u)$, showing at distances larger than the bond length, suggesting a non-negligible reorganization of the lone pairs prior to bond formation. Finally, we find that $\Delta h(u)$ is systematically larger than in $\mathrm{Li}_{2}$ and $\mathrm{F}_{2}$, as it corresponds to the formation of three electron pairs in $\mathrm{CO}$. Interestingly, $\mathrm{CO}$ and $\mathrm{F}_{2}$ have the shortest distance between the maximum and the minimum of $\Delta h(u)$ (see Table 1), indicating that these molecules experience a less drastic deformation of the electron-pair length upon bond formation. This fact is in agreement with the more electronegative character of the composing atoms, conferring them a lower capacity to be deformed.

A most interesting electron reorganization occurs in LiH ground and first excited states. ${ }^{85}$ The $\mathrm{X}^{1} \Sigma^{+}$and $\mathrm{A}^{1} \Sigma^{+}$states dissociate into $\mathrm{H}\left({ }^{2} S\right)+\operatorname{Li}\left({ }^{2} S\right)$ and $\mathrm{H}\left({ }^{2} S\right)+\operatorname{Li}\left({ }^{2} P\right)$, respectively. The adiabatic ground state, $\mathrm{X}^{1} \Sigma^{+}$, is dominated by a diabatic ionic state at the equilibrium but, as the molecule stretches, the PEC passes through an avoided crossing and the state is predominantly covalent in nature. In this sense, the character of the bond in $\mathrm{LiH}$ changes from covalent to ionic as the molecule is formed. This change of bond character is accompanied by an electron transfer from hydrogen to lithium, which is commonly known as the harpoon mechanism. This peculiar mechanism is given by the crossing between two diabatic states, the ionic and the lowest-lying covalent ones, around $3 \AA$. The $\mathrm{A}^{1} \Sigma^{+}$state is even more complicated because it results from the crossing of three diabatic states, the ionic and the two lowest-lying covalent diabatic states, giving rise to two avoided crossings (the first of which obviously is shared with the ground state). Hence, when the molecule is 
formed, the bonding character of $\mathrm{A}^{1} \Sigma^{+} \mathrm{LiH}$ changes from covalent to ionic and, then, back to covalent. The second avoided crossing takes places when $\mathrm{Li}$ and $\mathrm{H}$ are separated about $6 \AA$. The electron transfers occur in the regions close to the avoided crossings and were fully characterized in our previous work. ${ }^{85}$

The profile of $\Delta h(u)$ for $\mathrm{X}^{1} \Sigma^{+}$at equilibrium resembles $\mathrm{BH}$, peaking not far from the equilibrium distance. However, as we stretch the bond, one does not observe electron-pair depletion at short distances. In fact, it is only after we have passed the avoided crossing that we start to observe an increase of the probability at short electron-electron distances. In other words, the typical profile of covalent bond dissociation is only reproduced when we are in the part of the potential energy surface that is purely covalent. Although the profile of $\Delta h(u)$ for large $R$ is very similar for the ground and excited states, the situation at short bond lengths is reversed for the $\mathrm{A}^{1} \Sigma^{+}$state. As we approach the equilibrium distance, the profile does not reverse and we barely observe the formation of short-range electron pairs. This plot puts forward the rather polarized character of this bond, which is characterized by significant electron-pair stretching upon bond formation, i.e., completely opposite to all the molecules studied in this work. Even $\mathrm{HeH}^{+}$is a quite different case because in this molecule there was only internal reorganization of the electron pairs, which were never shifted to distances much larger than the bond length. 
$\mathrm{Li}_{2}$

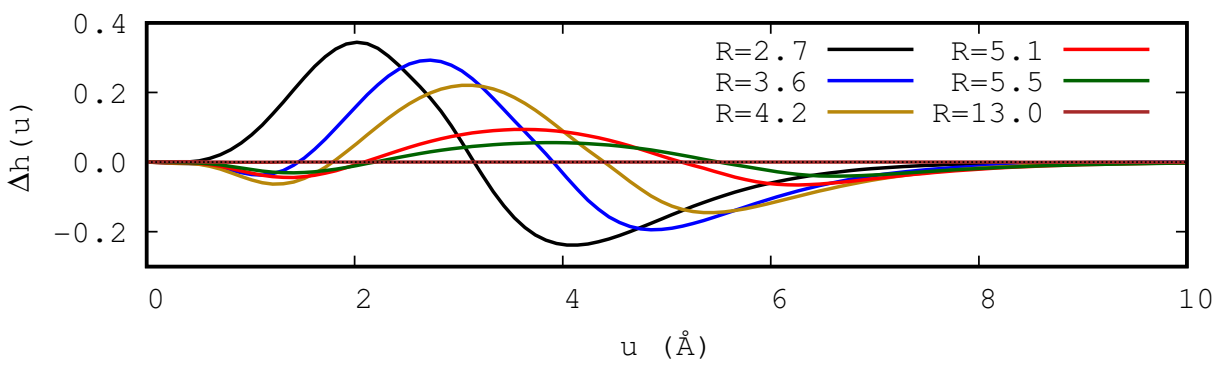

$\mathrm{CO}$
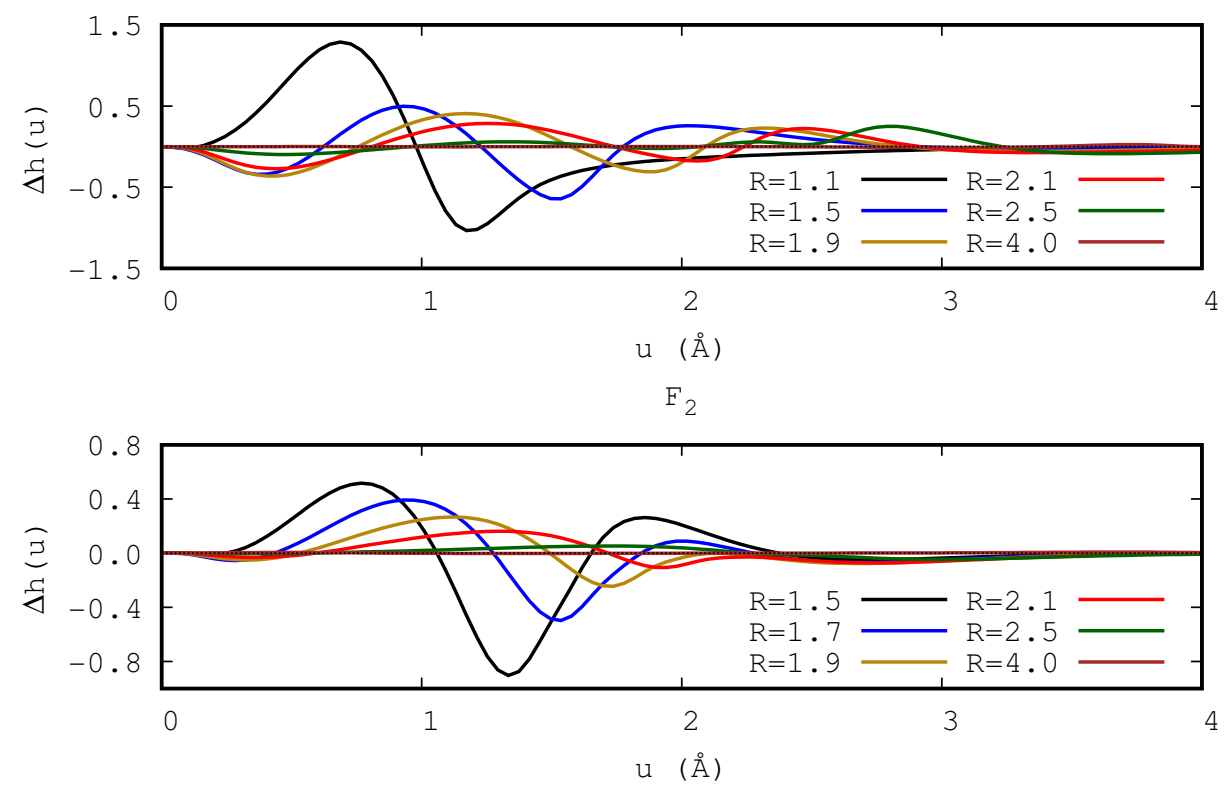

LiH $\left(X^{1} \Sigma^{+}\right)$

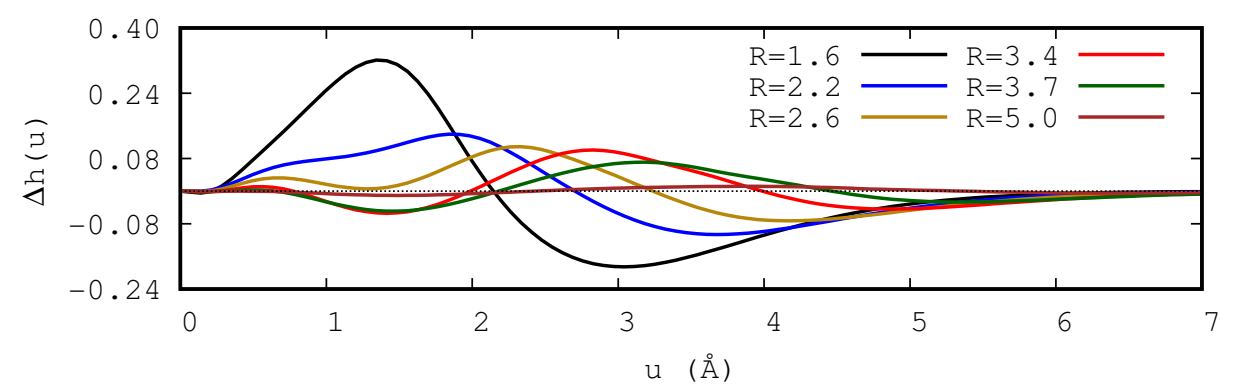

LiH $\left(A^{1} \Sigma^{+}\right)$

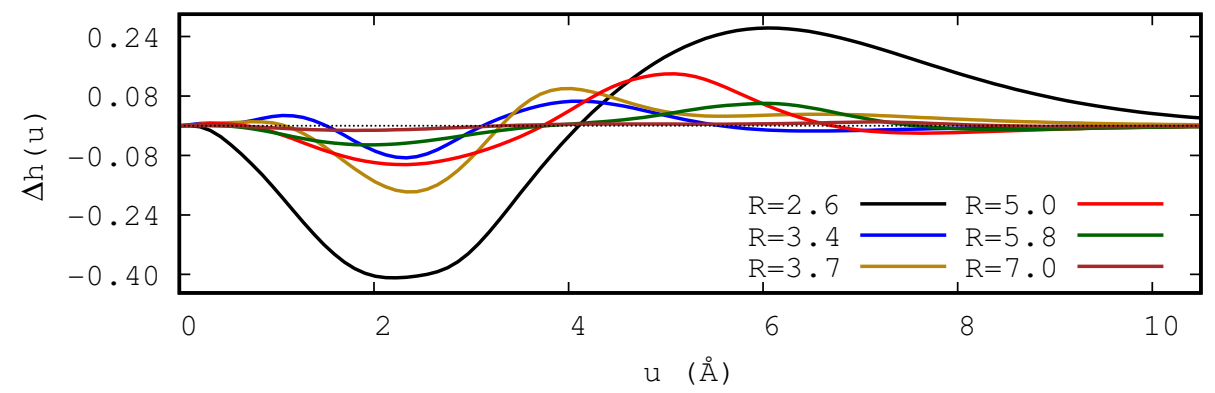

Figure 4: Relaxation holes for $\mathrm{Li}_{2}, \mathrm{CO}, \mathrm{F}_{2}$ and $\mathrm{LiH}$ at different bond lengths $(R)$. The ground $\left(X^{1} \Sigma^{+}\right)$and excited $\left(A^{1} \Sigma^{+}\right)$states of $\mathrm{LiH}$ were analyzed. The minimal bond length corresponds to the equilibrium geometry in all cases. All $R$ and $u$ in $\AA$. 


\section{Approximate Radial Intracule Densities}

In this section we assess the performance of approximate radial intracule densities in reproducing the plots of the previous sections. Thus far, we have employed CASSCF wavefunctions for $\mathrm{F}_{2}$ and $\mathrm{CO}$, and $\mathrm{FCI}$ for $\mathrm{H}_{2}, \mathrm{HeH}^{+}, \mathrm{BH}, \mathrm{Li}_{2}$ and $\mathrm{LiH}$ (both states). Both FCI and CASSCF yield $N$-representable 2-RDMs.

First of all, we replace the FCI/CASSCF calculation by CCSD for all the systems, except $\mathrm{CO}$, for which a multideterminant calculation is mandatory. Second, we use an approximate 2-RDM calculated from CCSD natural orbital occupancies. Namely, we apply the Müller approximation $^{66,67,77}$ using the energy-derivative CCSD 1-RDMs obtained from Gaussian. The latter are not $N$-representable and, thus, might present natural occupancies outside the physical range $[0,1]$. However, in the present cases, only a few populations did not meet this condition and the deviations from the occupation boundaries were small, producing no quantitative effect on the results presented.

We did not include $\mathrm{H}_{2}$ and $\mathrm{HeH}^{+}$because for these two-electron systems the CCSD wave functions actually correspond to the exact solution and, therefore, only the 2-RDM could be approximated. In addition, as we have just recently proven, the Müller approximation performs quite accurately in a weakly-correlated regime. ${ }^{53}$ Accordingly, our calculations on these systems confirm this finding, producing intracule plots that are indistinguishable from the exact ones and, therefore, we have omitted them in the manuscript. We have also omitted the excited state of $\mathrm{LiH}$. The approximate $\Delta h(u)$ plots for the rest of the molecules are plotted in Fig. 5. In all cases there is a very good agreement between the original calculations using FCI/CASSCF and the exact 2-RDM and these approximate wavefunctions using CCSD and Müller's approximation. The small difference occurs for the short-range part of $\Delta h(u)$ of $\mathrm{F}_{2}$, which can be attributed to the fact that CASSCF calculations did not include the $2 \sigma$ 
orbital in the active space whereas the CCSD wavefunction takes the correlation effects of this orbital into account. We also collect the information of the minima and the maxima of $\Delta h(u)$ in Table 2. Comparsion with the numbers in Table 1 reveals very small differences between the original and the approximate $\Delta h(u)$, validiting the use of the approximations to retrieve information about the electron pairing in electronic structures.

Table 2: First maximum $(M)$ and minimum $(m)$ of $\Delta h(u)$ for the systems analyzed with the approximate relaxation hole at the equilibrium distance (see Table 1). $u$ in $\AA$.

\begin{tabular}{ccccc}
\hline Molecule & $u_{M}$ & $u_{m}$ & $\Delta h\left(u_{M}\right)$ & $\Delta h\left(u_{m}\right)$ \\
\hline $\mathrm{BH}$ & 1.15 & 2.08 & 0.207 & -0.076 \\
$\mathrm{Li}_{2}$ & 2.04 & 4.10 & 0.353 & -0.241 \\
$\mathrm{CO}$ & 0.70 & 1.19 & 1.322 & -1.000 \\
$\mathrm{~F}_{2}$ & 0.80 & 1.35 & 0.657 & -0.970 \\
$\mathrm{LiH}\left(X^{1} \Sigma^{+}\right)$ & 1.36 & 3.03 & 0.325 & -0.184 \\
\hline
\end{tabular}


$\mathrm{Li}_{2}$
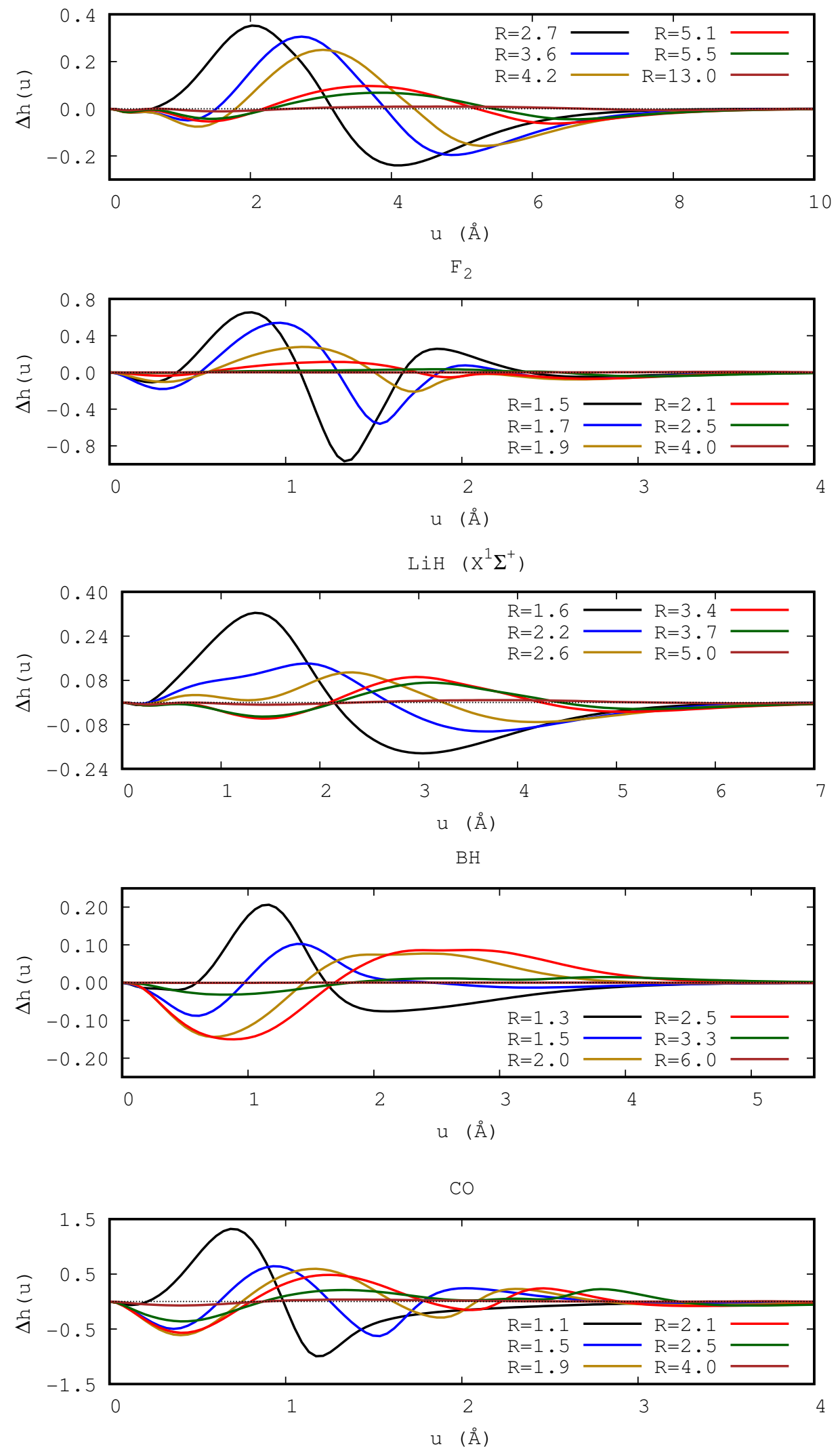

Figure 5: Approximate $\Delta h(u)$ for $\mathrm{Li}_{2}, \mathrm{~F}_{2}, \mathrm{LiH}, \mathrm{BH}$ and $\mathrm{CO}$ at different bond lengths $(R)$. The 2-RDM use the Müller approximation from CCSD $\left(\mathrm{Li}_{2}, \mathrm{~F}_{2}, \mathrm{BH}\right.$ and $\left.\mathrm{LiH}\right)$ and CASSCF (CO) wavefunctions. All $R$ and $u$ in $\stackrel{\AA}{\text {. }}$ 


\section{Conclusions}

In this paper we have studied the chemical formation process from the study of radial intracule densities. We have analyzed the relaxation holes, $\Delta h(u)$, resulting from the difference between the actual radial intracule density and the nonrelaxed one - constructed from atomic radial intracule densities and the pair density obtained from the overlap of the atomic densities. Our results show that the mechanism of electron-pair formation is contained in $\Delta h(u)$. In particular, the internal reorganization of electron pairs prior to bond formation and the covalent bond formation from electrons in separate atoms are completely recognizable processes from the shape of the relaxation hole, $\Delta h(u)$. The magnitude of $\Delta h(u)$, the shape of $\Delta h(u) \forall u<R_{e q}$ and the distance between the minimum and the maximum in $\Delta h(u)$ provides information about the nature of the chemical bond formed.

We have also suggested a computational affordable approach to calculate the radial intrac-

ule density from approximate pair densities and adequate wavefunctions such as CCSD or CASSCF as replacements of FCI in regimes of dynamic and nondynamic correlation, respectively. In all cases, there is a qualitative agreement with the reference calculation and, quite often, the relaxation holes produced from both methodologies are barely distinguishable. This approach paves the way to study electron-pair distributions in larger systems.

\section{Acknowledgments}

This research has been funded by the Spanish MINECO/FEDER Projects CTQ2014-52525P (E.M.), CTQ2014-54306-P (M.S.), CTQ2015-67608-P (X.L.), CTQ2015-67660-P (J.M.U.) and EUIN2017-88605 (E.M.), the Basque Country Consolidated Group Project No. IT58813, the Generalitat de Catalunya (Project 2014SGR931, Xarxa de Referència en Química 
Teòrica i Computacional, and the ICREA Academia 2014 prize (M.S.)). The FEDER grant UNGI10-4E-801 (European fund for Regional Development) has also supported this research. M.R.M. acknowledges the Spanish Ministry of Education, Culture and Sports for the doctoral grant FPU-2013/00176 and M.V.N. the Spanish Ministry of Economy, Industry and Competitiveness (MINECO) for the doctoral grant BES-2015-072734. The authors acknowledge the computational resources and technical and human support provided by the DIPC and the SGI/IZO-SGIker UPV/EHU.

\section{References}

(1) Marcelin, M. R. Contribution à l'étude de la cinétique physico-chimique. Annales of Physique 1915, 9, 120-231.

(2) Coulson, C. A. The electronic structure of some polyenes and aromatic molecules. VII. Bonds of fractional order by the molecular orbital method. Proc. Roy. Soc. A 1939, $169,413$.

(3) Mulliken, R. S. Electronic Population Analysis on LCAO-MO Molecular Wavefunctions. I. J. Chem. Phys. 1955, 23, 1833-1840.

(4) Daudel, R. Quantum Theory of The Chemical Bond; Reidel: Dordrecht, 1974.

(5) Bader, R. F. W. Atoms in Molecules: A Quantum Theory; Oxford Univ. Press: Oxford, 1990.

(6) Lewis, G. N. The Atom and the Molecule. J. Am. Chem. Soc. 1916, 38, 762-786.

(7) Bader, R. F. W.; Stephens, M. E. Fluctuation and correlation of electrons in molecular systems. Chem. Phys. Lett. 1974, 26, 445-449.

(8) Bader, R. F. W.; Stephens, M. E. Spatial localization of the electronic pair and number distributions in molecules. J. Am. Chem. Soc. 1975, 97, 7391-7399. 
(9) Fradera, X.; Austen, M. A.; Bader, R. F. W. The Lewis Model and Beyond. J. Phys. Chem. A 1999, 103, 304-314.

(10) Becke, A. D.; Edgecombe, K. E. A simple mesure of electron localization in atomic and molecular systems. J. Chem. Phys. 1990, 92, 5397-5403.

(11) Ponec, R.; Strnad, M. Population analysis of pair densities: A link between quantum chemical and classical picture of chemical structure. Int. J. Quant. Chem. 1994, 50, 43-53.

(12) Ponec, R. Electron pairing and chemical bonds. Chemical structure, valences and structural similarities from the analysis of the Fermi holes. J. Math. Chem. 1997, 21, 323333.

(13) Silvi, B. The Spin Pair Compositions as Local Indicators of the Nature of the Bonding. J. Phys. Chem. A 2003, 107, 3081-3085.

(14) Matito, E.; Solà, M.; Salvador, P.; Duran, M. Electron sharing indexes at the correlated level. Application to aromaticity calculations. Faraday Discuss. 2007, 135, 325-345.

(15) Matito, E.; Silvi, B.; Duran, M.; Solà, M. Electron localization function at the correlated level. J. Chem. Phys. 2006, 125, 024301.

(16) Francisco, E.; Martín Pendás, A.; Blanco, M. A. Electron number probability distributions for correlated wave functions. J. Chem. Phys. 2007, 126, 094102.

(17) Matito, E.; Solà, M. The role of electronic delocalization in transition metal complexes from the electron localization function and the quantum theory of atoms in molecules viewpoints. Coord. Chem. Rev. 2009, 253, 647-665.

(18) Kohout, M. A Measure of Electron Localizability. Int. J. Quant. Chem. 2004, 97, $651-658$. 
(19) Matito, E.; Poater, J.; Solà, M.; Duran, M.; Salvador, P. J. Phys. Chem. A 2005, 109, 9904-9910.

(20) Feixas, F.; Matito, E.; Duran, M.; Solà, M.; Silvi, B. Electron localization function at the correlated level: a natural orbital formulation. J. Chem. Theory Comput. 2010, 6, $2736-2742$.

(21) Ponec, R.; Cooper, D. L.; Savin, A. Analytic Models of Domain-Averaged Fermi Holes: A New Tool for the Study of the Nature of Chemical Bonds. Chem. Eur. J. 2008, 14, $3338-3345$.

(22) Löwdin, P.-O. Quantum theory of many-particle systems. I. Physical interpretations by means of density matrices, natural spin-orbitals, and convergence problems in the method of configurational interaction. Phys. Rev. 1955, 97, 1474-1489.

(23) Born, M. Quantenmechanik der stoßvorgänge. Zeitschrift für Physik 1926, 38, 803-827.

(24) Coulson, C. A.; Neilson, A. H. Electron correlation in the ground state of helium. Proc. Phys. Soc. London 1961, 78, 831-837.

(25) McWeeny, R. Some recent advances in density matrix theory. Rev. Mod. Phys. 1960, 32, 335-369.

(26) Bochicchio, R. C.; Ponec, R.; Lain, L.; Torre, A. On the Physical Meaning of Bond Indices from the Population Analysis of Higher Order Densities. J. Phys. Chem. A 1998, 102, 7176-7180.

(27) Ponec, R.; Roithova, J. Domain-averaged Fermi holes - a new means of visualization of chemical bonds. Bonding in hypervalent molecules. Theor. Chem. Acc. 2001, 105, $383-392$. 
(28) Blanco, M. A.; Martín Pendás, A.; Francisco, E. Interacting quantum atoms: a correlated energy decomposition scheme based on the quantum theory of atoms in molecules. J. Chem. Theory Comput. 2005, 1, 1096-1109.

(29) Francisco, E.; Martín Pendás, A.; Blanco, M. A. A molecular energy decomposition scheme for atoms in molecules. J. Chem. Theory Comput. 2006, 2, 90-102.

(30) Mayer, I.; Hamza, A. Energy decomposition in the topological theory of atoms in molecules and in the linear combination of atomic orbitals formalism: a note. Theor. Chim. Acta (Berlin) 2001, 105, 360-364.

(31) Salvador, P.; Duran, M.; Mayer, I. One-and two-center energy components in the atoms in molecules theory. J. Chem. Phys. 2001, 115, 1153-1157.

(32) Vyboishchikov, S. F.; Salvador, P.; Duran, M. Density functional energy decomposition into one-and two-atom contributions. J. Chem. Phys. 2005, 122, 244110.

(33) Tognetti, V.; Joubert, L. Density functional theory and Bader's atoms-in-molecules theory: towards a vivid dialogue. Phys. Chem. Chem. Phys. 2014, 16, 14539-14550.

(34) Salvador, P.; Mayer, I. Energy partitioning for "fuzzy" atoms. J. Chem. Phys. 2004, 120, 5046-5052.

(35) Ruiz, I.; Matito, E.; Holguín-Gallego, F. J.; Francisco, E.; Pendás, Á. M.; RochaRinza, T. Fermi and Coulomb correlation effects upon the interacting quantum atoms energy partition. Theor. Chem. Acc. 2016, 135, 209.

(36) Mayer, I.; Matito, E. Calculation of local spins for correlated wave functions. Phys. Chem. Chem. Phys. 2010, 10, 11308-11314.

(37) Ramos-Cordoba, E.; Matito, E.; Mayer, I.; Salvador, P. Toward a Unique Definition of the Local Spin. J. Chem. Theory Comput. 2012, 8, 1270-1279. 
(38) Ramos-Cordoba, E.; Matito, E.; Salvador, P.; Mayer, I. Local spins: improved Hilbertspace analysis. Phys. Chem. Chem. Phys. 2012, 14, 15291-15298.

(39) Ramos-Cordoba, E.; Salvador, P.; Piris, M.; Matito, E. Two new constraints for the cumulant matrix. J. Chem. Phys. 2014, 141, 234101.

(40) Thakkar, A. J.; Tripathi, A.; Smith Jr, V. H. Molecular x-ray-and electron-scattering intensities. Phys. Rev. A 1984, 29, 1108.

(41) Thakkar, A. J.; Tripathi, A.; Smith, V. H. Anisotropic electronic intracule densities for diatomics. Int. J. Quant. Chem. 1984, 26, 157-166.

(42) Watanabe, N.; Kamata, Y.; Yamaguchi, K.; Udawa, Y.; Miller, T. Calculation of X-ray scattering intensities by means of the coupled cluster singles and doubles model. J. Comput. Chem. 2001, 22, 1315-1320.

(43) Curl, R. F.; C. A. Coulson., C. A. Coulomb hole in the ground state of two electron atoms. Proc. Phys. Soc. 1965, 85, 647-652.

(44) Boyd, R. F.; Coulson, C. A. Coulomb hole in some excited states of helium. J. Phys. B: Atom. Molec. Phys. 1973, 6, 782-793.

(45) Boyd, R. J.; Sarasola, C.; Ugalde, J. M. Intracule densities and electron correlation in the hydrogen molecule. J. Phys. B: Atom. Molec. Phys. 1988, 21, 2555.

(46) Besley, N. A.; Gill, P. W. Atomic and molecular intracules for excited states. J. Chem. Phys. 2004, 120, 7290-7297 (and references therein).

(47) Fradera, X.; Duran, M.; Mestres, J. The relevance of the Laplacian of intracule and extracule density distributions for analyzing electron-electron interactions in molecules. J. Chem. Phys. 1997, 10\%, 3576-3583. 
(48) Fradera, X.; Duran, M.; Mestres, J. The mapping of the local contributions of Fermi and Coulomb correlation into intracule and extracule density distributions. J. Chem. Phys. 2000, 113, 2530-2543.

(49) Fradera, X.; Sarasola, C.; Ugalde, J. M.; Boyd, R. J. The topological features of the intracule density of the uniform electron gas. Chem. Phys. Lett. 1999, 304, 393-398.

(50) Ugalde, J. M.; Sarasola, C.; Dominguez, L.; Bovd, R. J. The evaluation of electronic extracule and intracule densities and related probability functions in terms of Gaussian basis functions. J. Math. Chem. 1991, 6, 51-61.

(51) Sarasola, C.; Ugalde, J. M.; Boyd, R. J. The evaluation of extracule and intracule densities in the first-row hydrides, $\mathrm{LiH}, \mathrm{BeH}, \mathrm{BH}, \mathrm{CH}, \mathrm{NH}, \mathrm{OH}$ and $\mathrm{FH}$, from selfconsistent field molecular orbital wavefunctions. J. Phys. B: Atom. Molec. Phys. 1990, 23, 1095 .

(52) Sarasola, C.; Dominguez, L.; Aguado, M.; Ugalde, J. The Laplacian of the intracule and extracule densities and their relationship to the shell structure of atoms. J. Chem. Phys. 1992, 96, 6778-6783.

(53) Rodríguez-Mayorga, M.; Ramos-Cordoba, E.; Via-Nadal, M.; Piris, M.; Matito, E. Comprehensive benchmarking of density matrix functional approximations. Phys. Chem. Chem. Phys. 2017, 19, 24029-24041.

(54) Via-Nadal, M.; Rodríguez-Mayorga, M.; Matito, E. A Salient Signature of van der Waals Interactions. Phys. Rev. A 2017, 96, 050501.

(55) Via-Nadal, M.; Rodríguez-Mayorga, M.; Ramos-Cordoba, E.; Matito, E. Singling out Weak and Strong Correlation. Phys. Rev. Lett. submitted.

(56) Piris, M.; Lopez, X.; Ugalde, J. Correlation holes for the helium dimer. J. Chem. Phys. 2008, 128, 134102. 
(57) Gori-Giorgi, P.; Seidl, M.; Savin, A. Intracule densities in the strong-interaction limit of density functional theory. Phys. Chem. Chem. Phys. 2008, 10, 3440-3446.

(58) Cioslowski, J.; Liu, G. Topology of electron-electron interactions in atoms and molecules. II. The correlation cage. J. Chem. Phys. 1999, 110, 1882-1887.

(59) Rodríguez-Mayorga, M.; Ramos-Cordoba, E.; Lopez, X.; Solà, M.; Ugalde, J. M.; Matito, E. The Coulomb Hole of the Ne Atom. submitted

(60) Mercero, J. M.; Valderrama, E.; Ugalde, J. M. In Metal-Ligand Interactions; Russo, N., Salahub, D. R., Witko, M., Eds.; Kluwer Academic Publishers: The Netherlands, 2003; pp 205-239.

(61) Mercero, J. M.; Rodríguez-Mayorga, M.; Matito, E.; Lopez, X.; Ugalde, J. M. The electron-pair density distribution of the ${ }^{1,3} \Pi_{u}$ excited states of $\mathrm{H}_{2}$. Can. J. Chem. 2017, 94, 998-1001.

(62) Piris, M.; Lopez, X.; Ugalde, J. M. Electron-pair density relaxation holes. J. Chem. Phys. 2008, 128, 214105.

(63) Eddington, A. S. Fundamental theory; Cambridge University Press: Cambridge, 1946.

(64) Ramos-Cordoba, E.; Salvador, P.; Matito, E. Separation of dynamic and nondynamic correlation. Phys. Chem. Chem. Phys. 2016, 18, 24015-24023.

(65) Ramos-Cordoba, E.; Matito, E. Local Descriptors of dynamic and nondynamic correlation. J. Chem. Theory Comput. 2017, 13, 2705-2711.

(66) Müller, A. M. K. Explicit approximate expression between reduced two- and oneparticle density matrices. Phys. Lett. 1984, 105A, 446-452.

(67) Buijse, M. A. Thesis: Electron Correlation. Fermi and Coulomb holes, dynamical and nondynamical correlation. Ph.D. thesis, Vrije Universiteit, Amsterdam, The Netherlands, 1991. 
(68) Helgaker, T.; Jorgensen, P.; Olsen, J. Molecular Electronic Structure Theory; Wiley: Chitester, 2000.

(69) Chávez-Calvillo, R.; García-Revilla, M.; Francisco, E.; Martín Pendás, A.; RochaRinza, T. Dynamical correlation within the Interacting Quantum Atoms method through coupled cluster theory. Comput. Theor. Chem. 2015, 1053, 90-95.

(70) Holguín-Gallego, F. J.; Chávez-Calvillo, R.; García-Revilla, M.; Francisco, E.; Martín Pendás, A.; Rocha-Rinza, T. Electron correlation in the interacting quantum atoms partition via coupled-cluster lagrangian densities. J. Comput. Chem. 2016, 37, $1753-1765$.

(71) Wang, Y. G.; Matta, C.; Werstiuk, N. H. Comparison of localization and delocalization indices obtained with Hartree-Fock and conventional correlated methods: Effect of Coulomb correlation. J. Comput. Chem. 2003, 24, 1720-1729.

(72) García-Revilla, M.; Francisco, E.; Costales, A.; Martín Pendás, A. Performance of the density matrix functional theory in the quantum theory of atoms in molecules. J. Phys. Chem. A 2012, 116, 1237-1250, ibid, 9216 (2012).

(73) Feixas, F.; Vandenbussche, J.; Bultinck, P.; Matito, E.; Solà, M. Electron delocalization and aromaticity in low-lying excited states of archetypal organic compounds. Phys. Chem. Chem. Phys. 2011, 13, 20690-20703.

(74) Piris, M.; Ugalde, J. Perspective on natural orbital functional theory. Int. J. Quant. Chem. 2014, 114, 1169-1175.

(75) Cioslowski, J.; Piris, M.; Matito, E. Robust validation of approximate 1-matrix functionals with few-electron harmonium atoms. J. Chem. Phys. 2015, 143, 214101.

(76) Pernal, K.; Giesbertz, K. J. H. Reduced Density Matrix Functional Theory (RDMFT) 
and Linear Response Time-Dependent RDMFT (TD-RDMFT). Top. Curr. Chem. 2015, 368, 125-183.

(77) Buijse, M. A.; Baerends, E. J. An approximate exchange-correlation hole density as a functional of the natural orbitals. Molec. Phys. 2002, 100, 401-421.

(78) Knowles, P. J.; Handy, N. C. A new determinant-based full configuration interaction method. Chem. Phys. Lett. 1984, 111, 315-321.

(79) Knowles, P. J.; Handy, N. C. A determinant based full configuration interaction program. Comput. Phys. Commun. 1989, 54, 75.

(80) Frisch, M. J. et al. Gaussian 09 Revision D.01. Gaussian Inc. Wallingford CT 2009.

(81) Matito, E.; Feixas, F. DMn program. 2009; University of Girona (Spain) and University of Szczecin (Poland).

(82) Feixas, F.; Solà, M.; Barroso, J. M.; Ugalde, J. M.; Matito, E. New Approximation to the Third-Order Density. Application to the Calculation of Correlated Multicenter Indices. J. Chem. Theory Comput. 2014, 10, 3055-3065.

(83) Rodríguez-Mayorga, M. RHO2-OPS: 2-DM Operations. 2016; Institute of Computational Chemistry and Catalysis, University of Girona, Catalonia, Spain.

(84) Cioslowski, J.; Liu, G. Fast evaluation of electron intracule and extracule densities on large grids of points. J. Chem. Phys. 1996, 105, 4151-4158.

(85) Rodríguez-Mayorga, M.; Ramos-Cordoba, E.; Salvador, P.; Solà, M.; Matito, E. Bonding description of the Harpoon Mechanism. Molec. Phys. 2016, 114, 1345.

(86) Martín Pendás, A.; Blanco, M. A.; Costales, A.; Mori Sánchez, P.; Luaña, V. Nonnuclear Maxima of the Electron Density. Phys. Rev. Lett. 1999, 83, 1930-1933. 
(87) Postils, V.; Garcia-Borrás, M.; Solà, M.; Luis, J. M.; Matito, E. Chem. Commun. 2015, $51,4865-4868$. 